\title{
Percolation with excluded small clusters and Coulomb blockade in a granular system
}

\author{
A. S. Ioselevich and D. S. Lyubshin \\ Landau Institute for Theoretical Physics RAS, 117940 Moscow, Russia, \\ Moscow Institute of Physics and Technology, Moscow 141700, Russia.
}

(Dated: October 29, 2018)

\begin{abstract}
We consider dc-conductivity $\sigma$ of a mixture of small conducting and insulating grains slightly below the percolation threshold, where finite clusters of conducting grains are characterized by a wide spectrum of sizes. The charge transport is controlled by tunneling of carriers between neighboring conducting clusters via short "links" consisting of one insulating grain. Upon lowering temperature small clusters (up to some $T$-dependent size) become Coulomb blockaded, and are avoided, if possible, by relevant hopping paths. We introduce a relevant percolational problem of next-nearest-neighbors (NNN) conductivity with excluded small clusters and demonstrate (both numerically and analytically) that $\sigma$ decreases as power law of the size of excluded clusters. As a physical consequence, the conductivity is a power-law function of temperature in a wide intermediate temperature range. We express the corresponding index through known critical indices of the percolation theory and confirm this relation numerically.

PACS numbers: 72.23.Hk, 73.22.-f, 72.80.Tm
\end{abstract}

A mixture of metallic and insulating grains is an important practical system appearing in numerous applications, such as ceramics, catalysts, and powders. It is also a useful model, describing many realistic composite materials (see [1]). Normally the properties of this system are described on the classical level: by the effective medium theory 2] (away from the percolation threshold), or by the percolation theory [3, 4] (near the threshold). For traditional "old-fashioned" mixtures (with the sizes of grains $a$ on the scale of microns), the classical approach is sufficient. However, in recent years a special attention was drawn to nanocomposite materials with characteristic $a \sim 10 \mathrm{~nm}[5]$. For such small grains essentially quantum effects should become important, especially on the insulating side of the percolation transition: tunneling through insulating grains and the Coulomb blockade effect (see, e.g., [ $[6])$.

In this paper, for definiteness, we consider a mixture of spherical grains of the same diameter $a$, the fraction $x$ of them being metallic, while the fraction $1-x$ being insulating (Fig:1). Every pair of adjacent conducting grains is supposed to establish a good electric contact with dimensionless conductance $G \gg 1$, but still the resistance $1 / G$ of such a contact is larger than the resistance of a conducting grain itself, so that the latter will be altogether neglected in what follows.

If one ignores the transport through insulating grains, then the macroscopic dc-conductivity $\sigma$ of the system identically vanishes below the percolation threshold (for $x<x_{c}$ ), so that the system undergoes a sharp metalinsulator transition (see [3, 4]). The infinite metallic cluster penetrating the entire system and carrying the dc current does not exist below the threshold: there are only finite metallic clusters embedded into the insulating matrix. The value of $x_{c}$ depends on the particular arrangement of the mixture. For example, for the regular densely packed 3d array of identical spheres (see Fig 1.
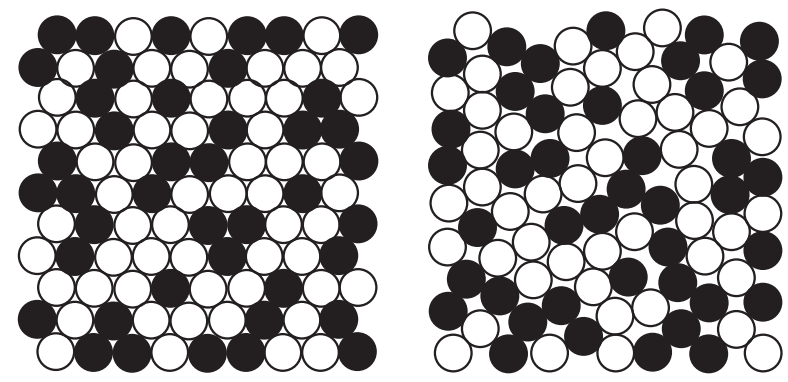

FIG. 1: A mixture of conducting grains (shown black) and insulating ones (white). Left panel: regular dense packing; right panel: topologically disordered random packing.

left panel) $x_{c} \approx 0.2[4]$, while for the $3 \mathrm{~d}$ random packing (right panel) $x_{c} \approx 0.35$ 7]. The critical exponents corresponding to the percolation transition are, however, universal and depend only on the dimensionality of the system $d$.

Thus, to obtain a finite conductivity for $x<x_{c}$ one should necessarily take into account processes in which electrons traverse insulating grains. Let $g$ be the dimensionless conductance of the shortest possible insulating bridge (consisting of a single insulating grain separating two conducting clusters, see Fig 2a). In what follows, we call these shortest bridges "links". Though usually $g$ is exponentially small, its finiteness smears the metalinsulator transition, and the macroscopic conductivity of the system below the threshold becomes nonzero.

If insulating grains are large and/or the temperature is relatively high, then electrons penetrate insulating grains due to activation mechanism (so that $g=g_{\text {act }} \propto$ $\exp \{-W / T\}$, where $W$ is the energy barrier for activation of a carrier in the insulator). For this incoherent mechanism the conductance $g^{(n)}$ of a bridge consisting 
(a)
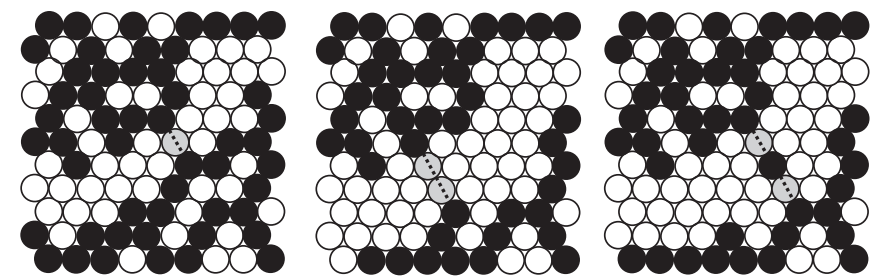

FIG. 2: Examples of paths (shown grey) through the insulator, connecting two large metallic clusters. (a) A singlegrain bridge (a link). The links remain effective connectors of metallic clusters even at lowest temperatures; (b) A twograin bridge. In the tunneling mode these and longer bridges do not contribute to the conductivity even at $T>E_{C}$; (c) A two-link chain path with an intermediate small (one-grain) metallic cluster. The paths involving small clusters are the first to be frozen out at $T<E_{C}$.

of $n$ insulating grains (see Fig $2 \mathrm{~b}$ for a $n=2$ bridge) is

$$
g^{(n)}=g_{\text {act }} / n,
$$

and all such bridges with not very large $n$ give comparable contributions to the effective conductance between two metallic clusters. Under the classical activation conditions the system is adequately described by a network made of randomly distributed classical conductances of two types: $G \gg 1$ and $g \ll 1$, and the percolation through only large conductances is established at $x=x_{c}$. The macroscopic conductivity $\sigma$ (measured in the units of $\left.\left(e^{2} / \hbar\right) a^{2-d}\right)$ of such a random network near the threshold is described by the formulas [4, [8]

$$
\begin{array}{ll}
\sigma_{\text {ins }}^{(0)} \sim g\left(x_{c}-x\right)^{-s}, & \Delta^{(0)} \ll x_{c}-x \ll 1, \\
\sigma_{\text {met }}^{(0)} \sim G\left(x-x_{c}\right)^{\mu}, & \Delta^{(0)} \ll x-x_{c} \ll 1,
\end{array}
$$

where $\mu$ is the universal conductivity exponent and $s$ is the universal dielectric permeability exponent (see Table 【i). Here

$$
\Delta^{(0)}=(g / G)^{1 /(\mu+s)}
$$

is the width of the crossover region where the contribution of the processes involving the insulating grains becomes comparable with the conductivity of the infinite cluster:

$$
\sigma_{\mathrm{ins}}^{(0)}\left(x_{c}-\Delta^{(0)}\right) \sim \sigma_{\mathrm{met}}^{(0)}\left(x_{c}+\Delta^{(0)}\right) .
$$

Within the crossover region

$$
\sigma_{\text {cross }}^{(0)} \sim g^{\mu /(\mu+s)} G^{s /(\mu+s)}, \quad\left|x_{c}-x\right| \lesssim \Delta^{(0)} .
$$

The formula (3) does not involve $g$, since the current manages to totally avoid links in this range of concentrations, so that the potential drops are distributed over the metallic infinite cluster, while the insulating component of the mixture does not play any role in dc-conductivity. Hence the classical formula (3) is valid for description of the mixture independent on the nature of the processes in the insulator. That is not the case for the formula (2) that describes the regime where the potential drops occur only on the insulating links between the metallic clusters, while the latter are essentially equipotential.

\begin{tabular}{||c||c|c|c|c|c|c|c|c|c||}
\hline$d$ & $\nu$ & $d_{f}$ & $\tau$ & $\mu$ & $\tilde{\zeta}$ & $s$ & $\theta$ & $\Theta$ & $\Theta^{\prime}$ \\
\hline \hline $\mathrm{d}=2$ & $4 / 3$ & $91 / 48$ & $187 / 91$ & 1.30 & 0.974 & 1.30 & 0.123 & 0.14 & 0.07 \\
\hline $\mathrm{d}=3$ & 0.875 & 2.524 & 2.32 & 2.14 & 1.3 & 0.74 & 0.28 & 0.38 & 0.28 \\
\hline
\end{tabular}

TABLE I: Numerical values of some critical exponents

For small grains and/or low temperatures, however, the tunneling mechanism dominates the transport via insulating grains, so that the conductance of the link is $g=g_{\text {tun }} \propto \exp \{-2 \kappa a\}$, where $\kappa$ is the tunneling decrement of the electronic wave function in the insulator [10]. In the tunneling mode the effective conductance of an insulating bridge of $n$ grains is

$$
g^{(n)}=A_{n} g_{\text {tun }}^{n}
$$

where $A_{n}$ is a model-dependent coefficient (typically $A_{n} \ll 1$ for $n>1$ ). An electron in this case has to tunnel through a sequence of $n$ insulating grains without any intermediate stop, and the corresponding tunneling amplitude exponentially decays with the length of the bridge.

Under the tunneling conditions the model of a network with two types of conductances is generally inapplicable. Instead of one smeared percolation transition at $x=x_{c}$, a cascade of smeared transitions at $x=x_{c}^{(m)}$ arises, $x_{c}^{(m)}$ being the threshold for percolation in the model where not only nearest neighbors but also next nearest and farther - up to $m$-th nearest neighbors - are assumed to be connected. In the range $x_{c}^{(m-1)}<x<x_{c}^{(m)}$ the conductivity $\sigma \sim g_{\text {tun }}^{(m)}$ is dominated by bridges of length $m$. The crossover between the adjacent ranges at $x=x_{c}^{(m)}$ is described by the formulas similar to (22), (3) with $g_{\text {tun }}^{(m)}$ standing for $g$ and $g_{\text {tun }}^{(m-1)}$ standing for $G$. Note that this cascade of smeared phase transitions has the same origin as the cascade-like concentration dependence of the temperature $T_{g}(x)$ of the spin-glass transition in those dilute magnetic alloys where the antiferromagnetic exchange interaction of localized magnetic ions exponentially decreases with distance [9].

Since we are interested in the case of very small grains and low temperatures, in the present paper we will adopt the tunneling scenario and concentrate on the behavior of $\sigma$ just below the principal threshold $x_{c}^{(1)} \equiv x_{c}$, in the insulator-dominated regime where the direct nearestneighbor $(\mathrm{NN})$ percolation via metal grains is impossible but the next-nearest-neighbor (NNN) percolation 
(which allows paths involving links, solitary insulating grains) still exists. Even without taking the Coulomb effects into account, the conductivity $\sigma_{\text {ins }}$ in the tunneling case should be somewhat suppressed compared to the classic percolational result (2): $\sigma_{\text {ins }}<\sigma_{\text {ins }}^{(0)}$. Indeed, in the tunneling regime the contributions to the conductance between metallic clusters coming from the insulating bridges with $n>1$ are suppressed, and only the contribution of links survives. Numerical simulations, however, demonstrate that $\sigma_{\text {ins }}$ differs from $\sigma_{\text {ins }}^{(0)}$ only in a somewhat smaller numerical coefficient (see below), while the index $s$ remains the same:

$$
\sigma_{\mathrm{ins}}(x)=c \sigma_{\mathrm{ins}}^{(0)} \sim g\left(x_{c}-x\right)^{-s}, \quad c<1 .
$$

This means that the problem with tunneling conductances remains in the same universality class as the standard one. The insensitivity of the index $s$ to suppression of insulating long bridges indicates that the intercluster conductance in the case of activation scenario is dominated by relatively short bridges of $n \sim 1$ insulating grains anyway, while long bridges of length $n \gg 1$ are irrelevant. Note that only the contribution of these very long bridges could be responsible for the difference in the values of $s$ for tunneling and activation cases (if there were any).

Slightly below the percolation threshold the system consists of finite metallic clusters characterized by a wide spectrum of numbers of grains $n_{i}$, sizes $r_{i}$, conductances $\mathcal{G}_{i}$, and charging energies $E_{i}$. The distribution function for clusters with $n$ grains is well known (see, e.g., [4]):

$$
N(n) \sim n^{-\tau} f\left(n / n_{c}\right), \quad n_{c} \sim \xi^{d_{f}}, \quad f(x) \sim e^{-x},
$$

where $d_{f}$ is the fractal dimension of the infinite cluster, and $\xi \sim a\left(x_{c}-x\right)^{-\nu} \gg 1$ is the correlation length. The clusters with the size $r \sim \xi$ and $n \sim n_{c}$ are commonly called critical clusters. The majority of grains belong to small clusters with $n \sim 1$ sites. The necessary exponents are given in the Table 1

In the range $1 \ll n \ll n_{c} \sim \xi^{d_{f}}$ the clusters are fractals, their physical characteristics are strongly fluctuating. However, one can estimate the corresponding average values for a fixed cluster size $n$.

An average dimensionless conductance across the cluster $i$ of fixed size $n$ in the range $1 \ll n \ll n_{c}$ can be estimated using standard expressions for conductivity of fractal objects (see [4]). The result is:

$$
\mathcal{G}(n) \equiv\left\langle\mathcal{G}_{i}\right\rangle_{n=\mathrm{const}} \sim G n^{-\tilde{\zeta} / d_{f}}, \quad \tilde{\zeta} \equiv 2-d+\mu / \nu .
$$

The clusters with $n_{i}<n_{\text {ext }}$, where

$$
n_{\mathrm{ext}} \sim G^{d_{f} / \tilde{\zeta}} \gg 1
$$

typically have large conductances $\mathcal{G}_{i}>1$ and can be treated as structureless point-like objects. Clusters with $n_{i}>n_{\text {ext }}\left(\right.$ and with $\mathcal{G}_{i}<1$ ) are, however, essentially extended objects. In this paper we consider only the case

$$
n_{\mathrm{ext}}>n_{c}
$$

when practically all existing clusters are point-like ones. The opposite case will be considered in a separate publication [11].

An average charging energy of clusters with fixed $n$ can be estimated from the scaling law for the average screened Coulomb interaction

$$
\langle U(r)\rangle \sim E_{C}^{(0)}(a / r)^{(\nu+s) / \nu},
$$

where

$$
E_{C}^{(0)} \sim e^{2} / a
$$

is the charging energy of a smallest one-grain cluster. The expression (12) is valid in the intermediate range $1 \ll r \ll \xi$ and gives a correct interpolation between the unscreened interaction $U(r) \sim E_{C}^{(0)} / r$ at $r \sim 1$ and the screened one $\langle U(r)\rangle \sim E_{C}^{(0)} / r \tilde{\epsilon}$ at $r>\xi$ (here $\tilde{\epsilon} \sim$ $\left(x_{c}-x\right)^{-s}$ is the static long-range dielectric constant of the system [8, 12]). As a result,

$$
E_{C}(n) \equiv\left\langle E_{i}\right\rangle_{n=\mathrm{const}} \sim E_{C}^{(0)} n^{-(\nu+s) / \nu d_{f}}
$$

For temperatures $T>E_{C}^{(0)}$ the Coulomb blockade effects are completely suppressed, and the expression (8), very similar to the classic result (2), is valid.

In the range $E_{C}^{(\mathrm{cr})}<T<E_{C}^{(0)}$ many clusters (those with $E_{i}>T$ ) are already frozen. The freezing condition may be rewritten as $n<\tilde{n}(T)$, where

$$
\tilde{n}(T) \sim\left(E_{C}^{(0)} / T\right)^{\nu d_{f} /(\nu+s)} .
$$

How does the conduction proceed in such a system? Clearly, an electron would prefer to travel via large clusters and to avoid small frozen clusters. Is the NNNpercolation still possible after the exclusion of small clusters with $n<\tilde{n}$ ? How does the exclusion affect the conductivity $\sigma$ ?

Let us call two metallic clusters "NNN-connected" if there is at list one link connecting these clusters (or, equivalently, at least one grain of the first cluster has a next nearest neighbor belonging to the second cluster). Now fix a certain $\tilde{n}$ and consider the "refined" system of all finite clusters with "large" sizes $n>\tilde{n}$ (see Fig $[3 \mathrm{~b}, \mathrm{c})$. If $\tilde{n} \gg \xi^{d_{f}}$ is too large, then only a few of such clusters will be NNN-connected, and there will be no "NNN-percolation" through the system. On the other hand, for $\tilde{n} \sim 1$ the NNN-percolation should definitely exist. We define $\tilde{n}_{c}$ to be the value of $\tilde{n}$ at which the NNN-percolation is first destroyed in the course of the refinement process. It is natural to expect

$$
\tilde{n}_{c} \sim n_{c} \sim \xi^{d_{f}}
$$


(a)

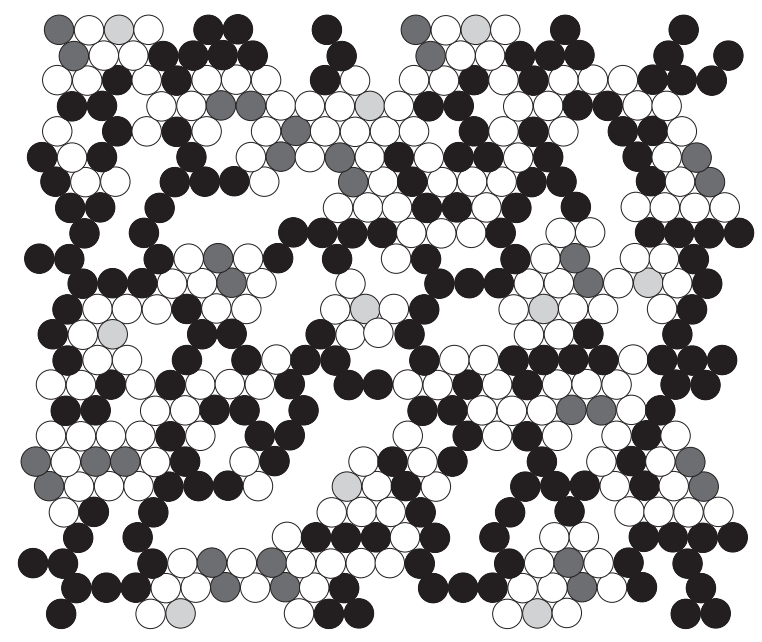

(b)

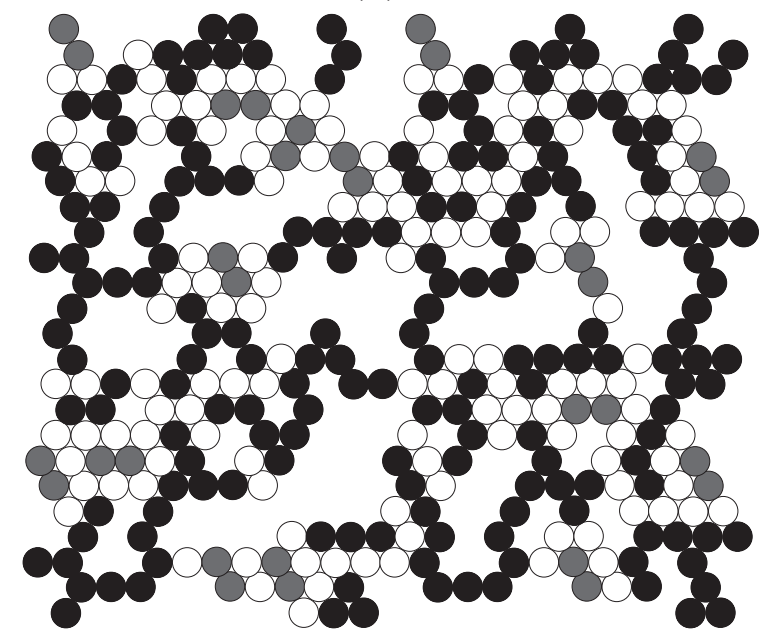

(c)

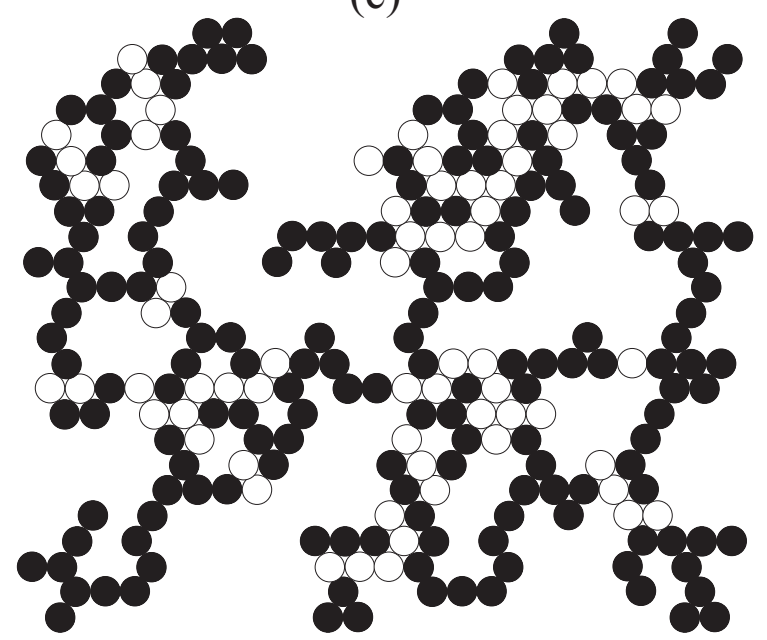

FIG. 3: An infinite network of NNN-connected clusters. The smallest clusters (consisting of one grain) are shown light grey; the second smallest ones (of two grains) are shown dark grey; all larger metallic clusters are shown black. (a) The full system, (b) The system with excluded smallest clusters, (c) The system with excluded both smallest and next smallest clusters. Note that NNN-percolation exists in all three cases.
On one hand, it is clear that $\tilde{n}_{c}$ can not be much larger than $n_{c}$, since the distance between so extraordinarily large clusters is exponentially large and they can not be NNN-connected. On the other hand, below we demonstrate that at $\tilde{n}_{c} \sim n_{c}$ the NNN-percolation still exists. Indeed, the average intercluster distance $R$ in the system of clusters with $n$ in the range $(1-\Lambda) n_{c}<n<n_{c}$ :

$$
R \sim\left[N\left(n_{c}\right) \Lambda n_{c}\right]^{1 / d} \sim \Lambda^{1 / d} \xi,
$$

is of the same order of magnitude as the size of critical clusters $\xi$. (For derivation of (18) we used the scaling relation $\tau-1=d / d_{f}$ between the critical exponents.) For $\Lambda>\Lambda_{\text {perc }} \sim 1$ there is an infinite network of interpenetrating clusters (interpenetration of two clusters $i$ and $j$ can be defined, say, as a condition $R_{i j}<\xi$ ). If $\Lambda-\Lambda_{\text {perc }}$ is not very small, then the fraction of critical clusters belonging to this interpenetrating network is of order unity.

To estimate the average number $N_{\text {links }}(\xi)$ of links NNN-connecting a typical pair of interpenetrating critical clusters, we use the following reasoning (very close to the arguments of Coniglio [13] used for determination of the fractal dimension of "red bonds"). Let us randomly choose some of the insulating sites (at concentration $\left.\Delta x \gtrsim x_{c}-x\right)$ and substitute conducting ones for them. Then the sample will be converted to a NNpercolating one with a similar value of correlation radius $\xi$. The initial critical clusters will become $\mathrm{NN}$-connected with probability of order unity and form the infinite cluster in this new system. On the other hand, typically two adjacent critical clusters become NN-connected when some insulating grain that was a critical link between these clusters is replaced by a conducting grain. The probability of such an event is $\sim \Delta x \cdot N_{\text {links }}(\xi)$. Of course, one can also imagine a situation when the NNconnection between these two clusters is established via a "chain bridge" involving one or more smaller clusters, but the corresponding probabilities are proportional to higher powers of $\Delta x$ and can be neglected. So, we conclude that the NN-connection of adjacent critical clusters is typically established when $\Delta x \cdot N_{\text {links }}(\xi) \sim 1$ and, consequently,

$$
N_{\text {links }}(\xi) \sim 1 /\left(x_{c}-x\right) \gg 1 .
$$

Thus, the interpenetrating critical clusters are typically multiply NNN-connected, and therefore the infinite network of interpenetrating critical clusters is NNNconnected.

Thus, if we exclude all clusters with $n<\tilde{n}(T)$, the NNN-percolation in the system of remaining clusters persists if $\tilde{n}(T)<n_{c}$, or, equivalently, if $T>E_{C}^{(\mathrm{cr})}$.

Although the conductivity $\sigma$ of a "refined" system of clusters with sizes $n>\tilde{n}$ is nonzero for $\tilde{n}<\tilde{n}_{c}$ (since the NNN-percolation is not destroyed), the magnitude of $\sigma$ is, 
of course, suppressed by the refinement. For $1 \ll \tilde{n}<\tilde{n}_{c}$ this suppression is strong:

$$
\sigma_{\mathrm{ins}}(x, \tilde{n}) \sim \sigma_{\mathrm{ins}}^{(0)}(x) \tilde{n}^{-\theta} .
$$

The new critical index $\theta$ can be related to the known ones:

$$
\theta=\frac{(d-2) \nu+s-1}{\nu d_{f}}
$$

To derive the relation (20) we note that at $\tilde{n} \sim n_{c}$ our system consists of critical clusters only, each pair of adjacent clusters being NNN-connected by $\sim N_{\text {links }}(\xi)$ links, so that we can estimate

$$
\sigma_{\text {ins }}\left(x, \tilde{n} \sim n_{c}\right) \sim g N_{\text {links }}(\xi) \xi^{2-d} .
$$

Then, comparing (21) with (19) at $\tilde{n} \sim n_{c}$, we immediately arrive at the relation (20). In two dimensions, making use of $\mu=s$ and Grassberger's precise result [14] $\mu / \nu=0.9826(8)$, we obtain $\theta=0.1227(4)$. Similarly, in $3 \mathrm{D}$ one has $\theta \approx 0.28$, which would be interesting to verify numerically. The index $\theta$ increases with $d$ and reaches $\theta=1 / 2$ at critical dimension $d=6$.

To verify the assumptions used in the above qualitative derivation, we performed moderate-statistics numerical computations for an ensemble of finite critical $2 \mathrm{~d}$ systems (at $x=x_{c}$ ) in the bus-bar geometry (see Fig (4). The sites adjacent to the two opposite sides of any sample of the ensemble belong to the conducting subsystem by definition, while other sites are conducting with probability $x_{c}$ and insulating with probability $1-x_{c}$. All the conducting clusters with $n<\tilde{n}$ are removed, but the barclusters, connected to the contacts, are "protected"they are never removed. The current is allowed to flow from the left bar to the right one via large clusters with $n>\tilde{n}$, eventually jumping from cluster to cluster through the links. One half of these systems (those with the two bars directly connected to each other, so that the current actually avoids links) is percolating and has very large conductance $\propto G$. The non-percolating systems of the other half have conductances $\propto g$ dominated by links. The expression for the size-dependent conductivity $\sigma_{\mathrm{cr}}$, averaged over the non-percolating half of ensemble, is

$$
\sigma_{\mathrm{cr}}(L, \tilde{n}) \sim g L^{s / \nu} \tilde{n}^{-\theta}, \quad 1 \ll \tilde{n} \ll L^{d_{f}},
$$

consistent with (19). The refinement process typically stops at $\tilde{n} \sim L^{d_{f}}$, when the size of maximal removed cluster reaches the systems size $L$ and only the two protected bar-clusters (NNN-connected by $\sim N_{\text {link }}(L)$ links) are left in the system (see Fig $4 \mathrm{~b}$ ).

Averages of finite conductivities $\sigma_{\mathrm{cr}}(L, \tilde{n})$ for a $L \times L$ square vs. cutoff parameter $\tilde{n}$ are given in Fig 5 , The data shown correspond to $L=256$ for bond problem and to $L=512$ for site problem. We used the Frank-Lobb (a)

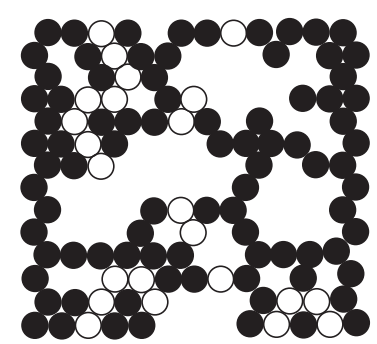

(b)

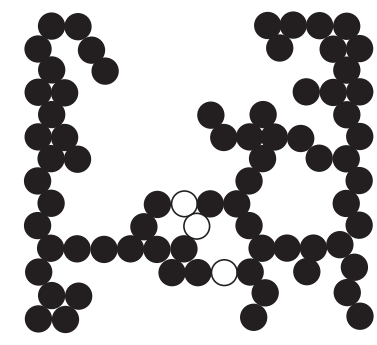

FIG. 4: A finite critical non-percolating system in the busbar geometry. (a) Before any refinement: all NNN-connected clusters and corresponding links (white circles) are shown. (b) After ultimate refinement: only the two bar-clusters and critical links between them are left.

algorithm 15] to collect statistics over about $2 \cdot 10^{4}$ samples per plot point. It is obvious that the deviations from power law (22) are small even for $\tilde{n} \sim 1$ or $\tilde{n}$ adjacent to the saturation plateau; standard fits yield $\theta=0.128(12)$ for the site variant and $\theta=0.126(10)$ for the bond variant, in good agreement with each other and with the theoretical prediction.

We also checked numerically that NNN percolation indeed shares the value $s$ with the ordinary NN problem. Scaling of $\sigma_{\mathrm{cr}}(L, \tilde{n})$ with sample size $L$ for $L=$ $16,32 \ldots 1024$ suggested $s / \nu=0.983(9)$ in perfect agreement with percolation value [14] $s / \nu=0.9826(8)$.

The very fact that $\theta \neq 0$, reliably confirmed above both analytically and numerically, already gives us an important insight into the physics of the NNN-conduction in an infinite system with large but finite $\xi$. A priori one could imagine two possible scenarios of the conduction process:

1. The conducting paths in a non-refined sample consist predominantly of largest critical clusters with size $n \sim n_{c}$, directly connected by critical links. Smaller clusters with $n \ll n_{c}$ are irrelevant. Were this scenario the actual one, the refinement process would not lead to any considerable suppression of the conductivity as long as $\tilde{n} \ll n_{c}$, and, consequently, one would expect $\theta=0$ and $s=$ $1-(d-2) \nu$.

2. Though the number of relevant conducting paths in a non-refined sample depends on $x$ and diverges as $x \rightarrow x_{c}$, each path consists predominantly of small clusters with size $n \sim 1$. When all clusters with $n<$ $\tilde{n}$ are removed from our system due to refinement, the leading role passes on to the smallest remaining clusters with $n \sim \tilde{n}$. For this scenario one expects $\theta>0$ and $s>1-(d-2) \nu$.

Our results definitely indicate that the second scenario, and not the first one, is real. Note that the opposition of the two concepts described above is reminiscent of the 


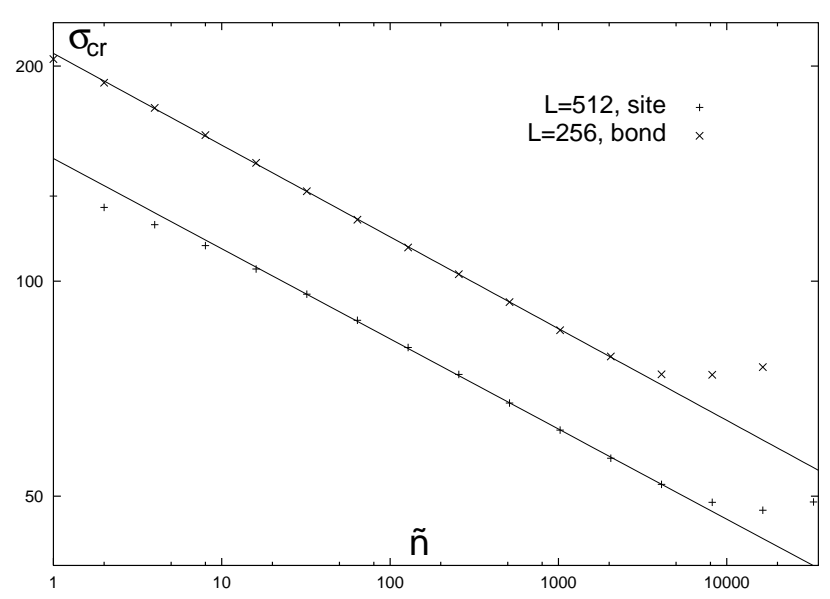

FIG. 5: Average conductivity of a $L \times L$ square as a function of the cutoff parameter $\tilde{n}$ for site and bond models. Lines correspond to fits with $\theta=0.128$ (bond) and $\theta=0.126$ (site).

alternative between the oversimplified "links and nodes" model [16] and a more sophisticated "links and blobs" model [17] in the standard NN-percolation problem .

Thus, substituting (15) into (19), for the temperature dependence of the conductivity we finally obtain

$$
\begin{aligned}
\sigma_{\text {ins }}(x, T) & \sim \sigma_{\text {ins }}(x, \tilde{n}(T)) \sim \sigma_{\text {ins }}^{(0)}(x)\left(T / E_{C}^{(0)}\right)^{\Theta}, \\
\Theta & =\frac{d_{f} \nu \theta}{\nu+s}=\frac{(d-2) \nu+s-1}{\nu+s},
\end{aligned}
$$

valid in the wide intermediate temperature range

$$
E_{C}^{(\mathrm{cr})} \ll T \ll E_{C}^{(0)},
$$

and not very close to the percolation transition (outside the critical crossover domain):

$\Delta(T) \ll x_{c}-x \ll 1, \quad \Delta(T)=\Delta^{(0)} \cdot\left(T / E_{C}^{(0)}\right)^{\Theta /(\mu+s)}$.

Within the critical crossover region (at $\left|x_{c}-x\right| \lesssim \Delta(T)$ ) the conductivity stops to depend on $x$ :

$$
\begin{aligned}
& \sigma_{\text {cross }}(x, T) \sim \sigma_{\text {cross }}^{(0)}\left(T / E_{C}^{(0)}\right)^{\Theta^{\prime}}, \\
& \Theta^{\prime}=\Theta \mu /(\mu+s) .
\end{aligned}
$$

The phase diagram for different regimes in the temperature-concentration plane is shown in Fig 6 .

At temperatures $T<E_{C}^{(\mathrm{cr})}$ the $T$-dependence of the conductivity becomes stretched exponential; it is due to the fact that at these low temperatures $\tilde{n}(T)>n_{c}$ and the current has no chance to avoid the Coulomb blockaded clusters. The underlying physics is very different from the exclusion of the clusters considered above; it is discussed in a separate publication [11].

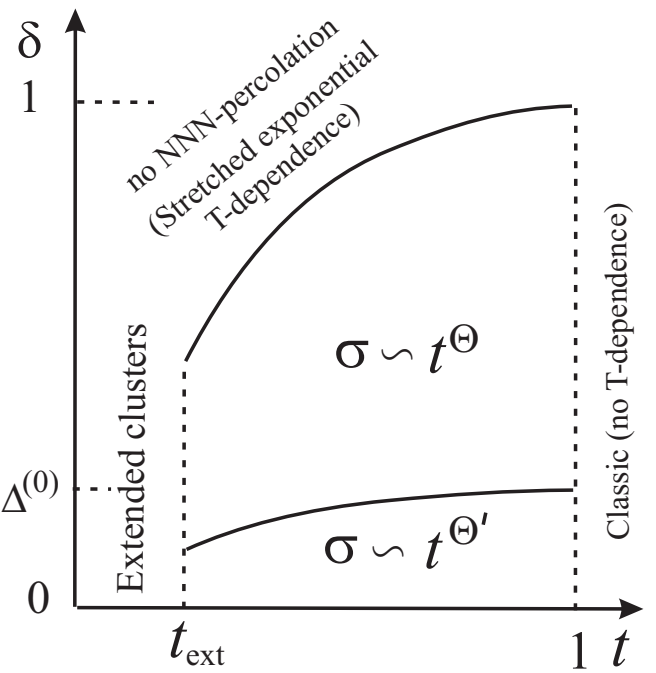

FIG. 6: Domains of the power-law $T$-dependence on the $\delta-t$ plane. Here $\delta \equiv x_{c}-x$ is the distance from the percolation transition, $t \equiv T / E_{C}^{(0)}$ is the dimensionless temperature, $t_{\text {ext }} \sim G^{(\nu+s) /[(2-d) \nu+\mu]} \ll 1$. The upper crossover line is given by $\delta \sim t^{2 /(\nu+s)}$, the lower one-by $\delta \sim \Delta^{(0)} t^{\Theta /(\mu+s)}$.

In conclusion, we have demonstrated that the Coulomb blockade effect in a granular material close to the percolation threshold leads to the power-law temperature dependence of conductivity, valid in a wide intermediate temperature-range. This dependence arises due to the wide power-law distribution of the charging energies of conducting clusters, characteristic for the critical region near the percolation threshold. The corresponding exponent is universal, it depends only on the dimensionality of the system and can be expressed through known critical exponents of the percolation theory. It is interesting to note that in three dimensions both exponents $\Theta=0.38$ and $\Theta^{\prime}=0.28$ are close to the exponent $1 / 3$ used for the fitting of the experimentally observed critical $T$-dependence of conductivity of thick amorphous (but, presumably, not granulated) films of $\mathrm{InO}$ and $\mathrm{TiN}$, in which the superconductivity was suppressed by strong magnetic field [18]. It is possible that for the latter systems the similar power law $\sigma(T)$-dependence is due to the self-induced inhomogeneity of disordered systems developing at low temperatures when the system approaches the MIT (see [19]).

The Authors are indebted to M.V.Feigelman and V.F.Gantmakher for valuable discussions of physics of granular metals. This work was supported by RFBR grant 06-02-16533.

[1] Disorder and Granular Media, eds. D. Bideau and A. Hansen, (North-Holland, Amsterdam, 1993)

[2] S. Kirkpatrick, Rev. Mod. Phys., 45, 574 (1973); 
D. S. McLachlan, M. Blaszkiewicz, and R. E. Newnham, J. Am. Ceram. Soc. 73, 2187 (1990)

[3] D. Stauffer and A. Aharony, Introduction to Percolation Theory (Taylor and Fransis, London, 1994)

[4] A. Bunde and S. Havlin, Percolation I, in Fractals and Disordered Systems, eds. A.Bunde and S. Havlin (Springer, Berlin, 1996)

[5] Nanocomposite Science and Technology, edited by P. M. Ajayan, L. S. Schadler, and P. V. Braun, (WileyVCH, Weinheim, 2003)

[6] Single Electron Tunnelling, edited by H. Grabert and M. H. Devoret (Plenum Press, New York and London, 1992)

[7] M. J. Powell, Phys. Rev. B21, 3725 (1980); D. Bouvard and F. F. Lange, Acta metall. mater, 39, 3083 (1991)

[8] A. L. Efros and B. I. Shklovskii, Phys. Stat. Sol. b 76, 475 (1976)

[9] M. Escorne and A. Mauger, Phys. Rev. B25, 4674 (1982); M. A. Novak, O. G. Symko, D. J. Zheng, and S. Ozerov, J. Appl. Phys. 57, 3418 (1985)

[10] In general, the conductance $g_{\text {tun }}$ depends on the geometry of the contact (e.g., on the angle between the two bonds formed by the two "terminal" conducting grains and the intermediate insulating grain). We neglect this dependence, since it does not affect the universal characteristics of the process.

[11] M. V. Feigelman and A. S. Ioselevich, to be published

[12] B. I. Shklovskii and A. L. Efros Electronic Properties of Doped Semiconductors, Springer, Berlin, (1984)

[13] A. Coniglio, Phys. Rev. Lett. 62, 3054 (1989)

[14] P. Grassberger, Physica A 262, 251 (1999)

[15] D. J. Frank, C. J. Lobb, Phys. Rev. B37, 302 (1988)

[16] A. S. Skal and B. I. Shklovskii, Sov. Phys. - Semiconductors, 8, 1586, (1974); P. G. de Gennes, J. de Physique 37, L1 (1976)

[17] H. J. Herrmann, H. Stanley, Phys. Rev. Lett. 53, 1121 (1984)

[18] V. F. Gantmakher, M. V. Golubkov, V. T. Dolgopolov, A. A. Shashkin, and G. E. Tsydynzhapov, JETP. Lett. 68, 363 (1998) and JETP. Lett. 71, 473 (2000); T. I. Baturina, D. R. Islamov, J. Bentner, C. Strunk, M. R. Baklanov, and A. Satta, JETP. Lett. 79, 337 (2004)

[19] D. Kowal and Z. Ovadyahu, Solid State Commm., 90, 783 (1994); M. V. Feigelman, A. S. Ioselevich, and M. A. Skvortsov Phys. Rev. Lett., 93, 136403 (2004); M. A. Skvortsov and M. V. Feigelman Phys. Rev. Lett., 95, $057002(2005)$ 